\title{
Fuzzy Emotion Recognition Using Semantic Facial Features and Knowledge-based Fuzzy
}

\author{
Dewi Yanti Liliana $^{1}$, T. Basaruddin ${ }^{2}$, M. Rahmat Widyanto ${ }^{3}$ \\ ${ }^{1} \mathrm{PhD}$ Candidate at Faculty of Computer Science, Universitas Indonesia, Indonesia \\ ${ }^{1}$ Lecturer at State Polytechnic of Jakarta, Indonesia \\ '1ewiyanti.liliana@tik.pnj.ac.id \\ ${ }^{2,3}$ Faculty of Computer Science, Universitas Indonesia, Indonesia, \\ ${ }^{2}$ chan@cs.ui.ac.id
}

\begin{abstract}
Automatic emotion recognition based on facial expression has been carried out by many researchers in computer vision field, mostly to classify six basic emotions defined by psychologist expert Ekman: angry, sad, fear, happy, disgust, and surprise. The problem is that in real situations, emotional experiences are not strictly separated into six different classes of emotions. Whereas a single emotion can naturally elicit other emotions simultaneously, e.g. being sadly angry at the same time. This study proposes a fuzzy emotion recognition as a mixture of basic emotions which is a natural characteristic of human emotion. Fuzzy emotion utilizes semantic facial features and psychologist expert knowledge extracted into a fuzzy inference engine. Semantic facial feature is a high-level feature in facial image which describes the condition of facial components, e.g. eyes is open, nose is wrinkle, and so on. The performance of the proposed system was tested on the own-made Indonesian Mixed Emotion Dataset (IMED) which yielded a promising result with accuracy rate $88.519 \%$, precision $88.519 \%$, and recall $\mathbf{8 9 . 0 1 1 \%}$. Furthermore, the implementation of the proposed work possibly enhances the real human and machine interaction in many applications.
\end{abstract}

Keyword: fuzzy emotion, geometric features, knowledge-based fuzzy, mixed emotion, natural emotion recognition, semantic facial features

\section{INTRODUCTION}

Emotion is a natural instinct that describes the condition of one's feelings in relation with the surrounding environment. Moreover, basic emotion is a fundamental emotion that has a separate characteristic and significantly differ from one another [1]. The classification of basic emotion was carried out by psychologist Ekman as a result of his long-term study throughout the world which mentioned six types of universal basic emotions: angry, sad, fear, happy, disgust, and surprise [2]. This is then used as a reference for many studies including research in the field of computer vision and machine learning, to classify six basic classes of emotions. Also, various facial expressions datasets with annotated basic emotion were developed such as CK+, JAFFE, DISFA, MUG, and many others [3]-[5] to help the analysis of the recognition performance. But in real situations, emotional experiences and facial expressions are not strictly separated into different emotion classes. This is confirmed based on Ekman's statement that even though six basic types of emotions are universal, there are individual variations in displaying facial expressions [6]. Three factors that cause individual variations in facial expressions are: 1) static facial signals that distinguish the emotion expression of each person, such as the location of cheekbones, eye shape, eyebrow shape, etc.; 2) personal experience and; 3) personal display rule which is a characteristic of how an individual expresses emotion. From this explanation it can be deduced that in real situation emotion in facial expression is not absolutely separated, but rather ambiguous between individuals.

Based on psychological analysis, there is a relationship between universal basic emotions with variations in individual characteristics in expressing these types of emotions. Moreover, beside basic emotion there is also mixed emotion performed by human in a daily life. Whereas existing researches simplify and neglect real factors in emotional expression by classifying basic emotions absolutely. Our proposed research is developing fuzzy emotion recognition framework as a generalization of mixed emotion. Fuzzy emotion recognition is the automatic emotion recognition from facial expression which complies with the principles of psychologists in investigating emotions by focusing on changes in facial components as well as accommodating the aspect of ambiguity and vagueness in facial expressions. Fuzzy emotion summarizes different human perceptions and tolerates uncertain emotion values, unlike many other classifiers including machine learning classifier such as Support Vector Machine (SVM) [7] and Deep Learning-based classifier [8] which work by classifying discrete classes of basic emotions. Moreover, differences in the characteristics of facial expressions can be tolerated by a fuzzy knowledge-based system. Thus, the proposed framework can adapt with different degrees and intervals of facial expressions and produce fuzzy emotion recognition results. We utilize a novel-semantic facial features to 
capture the facial component changes which form the facial expression. Semantic facial feature is a high-level semantic object feature which is extracted from facial landmarks. This high-level feature has a linguistic interpretation and it is understandable by human (e.g. eyes is open, mouth is closed, and so on), unlike other facial features based on texture or appearance which are only understandable by machine.

Our research contribution are as follows. First, we propose a fuzzy emotion model which intuitively and naturally mimics the way humans recognize multiple emotions in facial expressions. Second, we propose a semantic object level features as a representation of facial components through geometric facial feature extraction. Third, we acquire the psychologist knowledge of emotion from facial expression and conceptualize it in a rule-based fuzzy inference engine. Furthermore, we also develop facial expression dataset to measure the performance of the proposed framework. This dataset consists of several poses of many variations of mixed emotion as a combination of basic emotions. Furthermore, our own-made dataset: Indonesian Mixed Emotion Dataset (IMED) has been validated by a group of psychologists to ensure the reliability to become a ground truth dataset. We believe that this publicly available dataset will enlarge the existing facial expression dataset. The ultimate goal of the proposed research is to develop an artificial intelligence system to recognize real emotions that can be used for more real human and machine interactions and has a potential implementation in many fields such as psychology, education, health, and entertainment. We explain our novelty and the related work on the next section, followed by the implementation issues which are discussed into three sections. We test and measure the performance of our proposed work and discuss the result on a separate section. The last section gives a comprehensive conclusion of our research.

\section{RELATED WORK}

Emotion recognition is an active research challenge in the domain of Social Signal Processing (SSP) [9]. Many studies proposed different methods as well as different nonverbal cues as a social signal to recognize emotion; from facial expression, eye gaze, vocal intonation, and gesture [10]. Amongst all, facial expression is a nonverbal cue which gives more information about human emotion [11]. Many existing researches use facial expressions to recognize basic emotion [12]-[15]. Basic emotion recognition becomes popular in the community based on assumption that basic emotion classes have clear and distinguished characteristics. Meanwhile, less effort has been made on another type of emotion, or mixed emotion class. There are several existing studies in the literature which utilized various methods for basic emotion recognition, such as multilayer neural network [16], multi-kernel SVM [17], and convolutional neural network (CNN) [18], [19]. Those researches were reporting a very good performance. The problem is, human real emotions are not limited to basic emotion types only. This remains an open challenge, to recognize human real and natural emotions, in terms of mixed emotion.

Mixed emotions are defined as an affective experiences which activating two or more basic emotions, usually in opposite valences such as happy and sad [20]. Another name for this emotion class is complex emotion, multiple emotion, blended emotion, compound emotion, and so on. Compound emotion recognition as a combination of two different basic emotions in a static image was proposed by $\mathrm{Du}$ (2014) using Support Vector Machine classifier [21]. Another work for mixed emotion recognition in a temporal image was done by Liliana (2017) using Conditional Random Fields [22]. Unfortunately, the limitation of those works are in capturing real emotions which were limited only to two basic emotions in a single occurrence in Du [21], and a single basic emotion in each image frame which formed a sequence of mixed emotion in Liliana [22]. The novelty of this proposed research is to overcome the limitation of the existing studies in recognizing human natural emotion as a combination of multiple basic emotions. Fuzzy emotion framework recognizes the occurrence of multiple emotions in a facial expression image as well as describes the intensity of each emotion involved. To the best of our known, this approach has not been proposed in anywhere. The existing fuzzy approaches were using fuzzy logic as a basic emotion classifier [23]-[25], but not in recognizing a real fuzzy emotion. This is different from the proposed research, where fuzzy knowledge-based is used for fuzzy emotion recognition to show the fuzzy emotions and the fuzzy degrees of each emotion contained in a face image. This is conceptually distinguishing between existing research and the proposed research.

Feature extraction has an important role in classification process. Facial feature selection is a mechanism to provide a meaningful representation of a facial expression image. Several feature extraction techniques were used for facial expression recognition, most of them were based on facial shape [26], appearance [27], or hybrid features which combined shape and appearance features [13],[15]. Active Appearance Model (AAM) is a very well-known template-based method that combines shape and appearance features to produce facial landmarks as an important facial features [28]. This research utilizes AAM to detect facial important points in an input image which results a set of facial points in a cartesian coordinates [29]. The next section describes our proposed work, the fuzzy emotion recognition framework. 


\section{Proposed Work}

Generally, three stages for emotion recognition are: facial area detection, facial feature extraction, and emotion recognition [30]. We adopt those stages and depict our proposed work process diagram in Fig. 1.

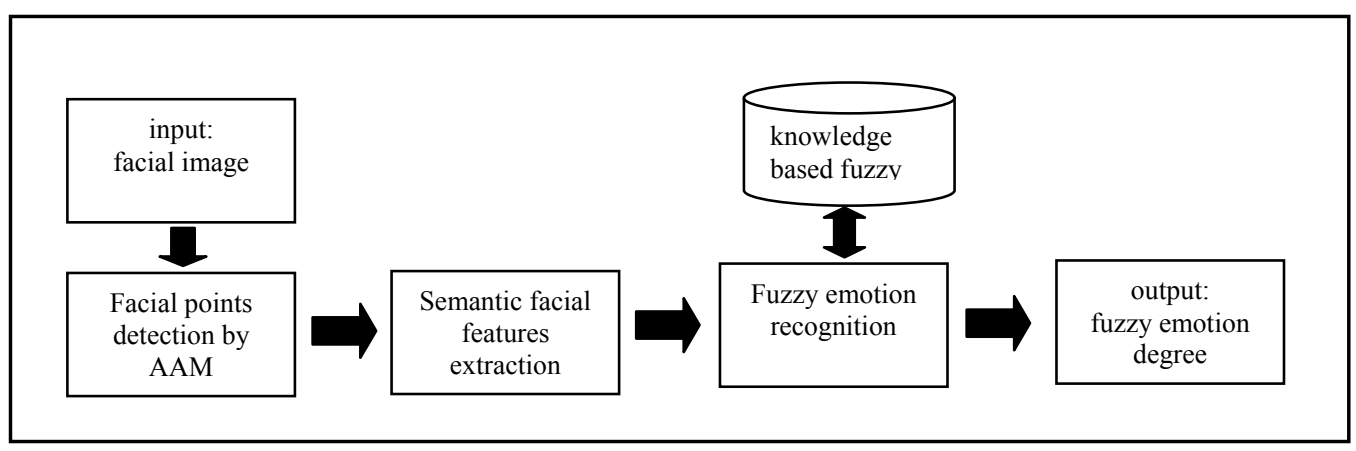

Fig. 1. Stages of fuzzy emotion recognition system

As depicted in Fig. 1, the initial stage after the input is processed into the system is facial points detection by AAM. The system utilizes AAM framework proposed by [29] to detect the important facial points which forms a face shape. This face shape is decomposed into several facial components i.e. eyebrow, eyes, nose, and mouth which are passed through the next stage for facial feature extraction. In the semantic facial feature extraction stage, facial component features are extracted using geometric calculation to result facial feature values. These values are then transformed into a semantic facial feature which has two values: linguistic of facial components and fuzzy value of facial features. The next stage is fuzzy emotion recognition which takes semantic facial feature values as input and produces the fuzzy emotion degrees from the fuzzy emotion knowledge-based which is synthesized from the psychologist knowledge on emotion recognition.

In this work, our own made facial expression dataset or Indonesian Mixed Emotion Dataset (IMED) is used to measure the recognition performance of the proposed system since no dataset for mixed emotion exists for public. IMED has been annotated by psychologists and is publicly available at http://imed.cs.ui.ac.id. This dataset consists of 15 actors who demonstrate various types of posed mixed emotion as well as basic emotions, as instructed by the director. Actors are Indonesian people from various ethnicities and ages. The postprocessing phase separated these videos into sequence of image frames. Fig. 2. depicts the IMED actors and their facial expressions. The next section will describe the semantic facial feature extraction process.

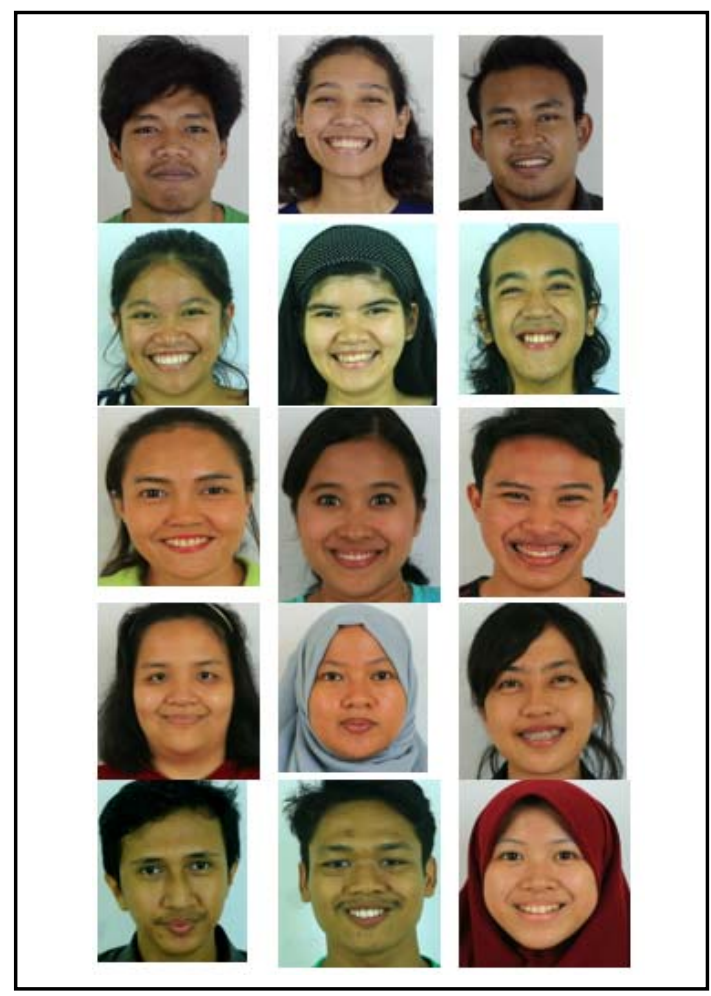

Fig. 2. Actors performing facial expressions in IMED dataset 


\section{SEMANTIC FACIAL FeATURES EXTRACTION}

Semantic facial feature is more meaningful than low-level feature (e.g. shape or texture) or the ordinary facial component features (e.g. eyes or mouth), since each facial component may have different condition. Whereas semantic facial feature describes the condition of facial component in a linguistic that is understandable by human. As an example, eye has three condition: narrow, normal, and wide. Hence, semantic facial feature is better representing the facial component's state (e.g. eye is wide). Facial points detection is a preliminary step prior to the semantic facial features extraction. AAM tools is used to produce the facial points locations.

\section{A. Active Appearance Model for Facial Points Detection}

Active Appearance Models (AAM) is a template matching model based on statistical methods which captures facial points [31]. AAM combines the shape and texture features in an image, resulting facial landmarks consists of several points. FPs form facial components that can be used for feature extraction purpose. We use the AAM implementation by [29] in a facial image which yields 68 facial points (FP) coordinates. Fig. 3 shows the FPs of an AAM template model.

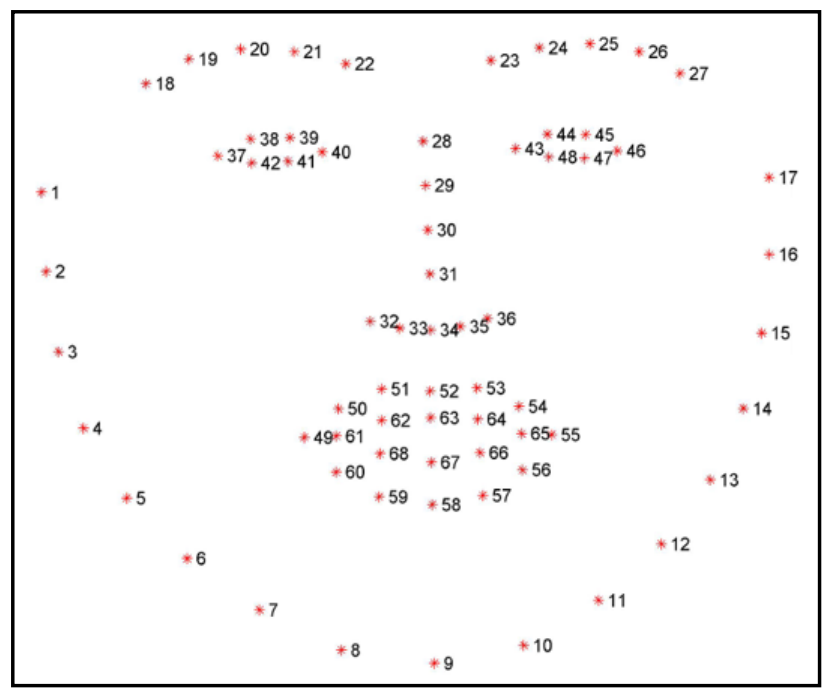

Fig. 3. The visualization of AAM facial points in facial image

\section{B. Geometric Facial Features}

Semantic facial features extraction is based on geometric facial features which utilizes the location of important points on the face in the calculation. This geometric feature is simple and low computation cost is needed since it works directly on a pixel basis [32], unlike appearance-based feature which works on the image area and involves a numerous data which needs more computational cost. Four facial components are used: eyebrows, eyes, nose, and mouth obtained from the FPs of the AAM result. So, all FPs in Fig. 3 are used except FPs on jaw area (FP1-FP17). Two types of geometric facial features are defined on these facial components, ellipse eccentricity and distance ratio.

Ellipse eccentricity describes how curve an ellipse object is. Facial components which have an elliptic (or half elliptic) shapes are eyebrows, eyes, and mouth. The formula for eccentricity is shown in the equation (1).

$$
e=c / a
$$

where $e$ denotes the eccentricity feature and $c$ denotes a square root of $a^{2}-b^{2} . a$ is the major axis and $b$ is the minor axis of an ellipse. $a$ is a half-width of facial components and $b$ is the half-height of it, applied to eyebrows, eyes and mouth. Meanwhile, the second geometric feature or distance ratio is obtained by dividing height to width, applied on eyebrows, eyes, nose, and mouth. We refer the geometric feature extraction process to our previously published reference [32]. For each facial component, the result of geometric facial features extraction is a set of feature vector which then being processed in a fuzzy facial inference system to gain the semantic facial features.

\section{Semantic Facial Features Generation Using Fuzzy Inference Engine}

Geometric features are passing through the semantic fuzzy facial inference engine. This engine is based on facial component rules which produces the linguistic condition (or semantic) of the facial component as well as the fuzzy value of it. Fuzzy Mamdani method is applied as the inference engine and the defuzzification is based on center of gravity method. Table 1 describes the input and output linguistic variables of the fuzzy facial inference engine, where each input has three membership functions: low, medium, and high. 
TABLE I. Semantic facial features from fuzzy inference engine

\begin{tabular}{|c|c|c|c|c|}
\hline $\begin{array}{c}\text { Facial } \\
\text { Component }\end{array}$ & $\begin{array}{c}\text { Input } \\
\text { (geometric features) }\end{array}$ & \multicolumn{2}{|c|}{ Output (semantic interpretation) } \\
\hline \multirow{2}{*}{ Eyebrows } & eccentricity & lower & normal & raise \\
\cline { 2 - 3 } & distance ratio & & & \\
\hline \multirow{2}{*}{ Eyes } & eccentricity & narrow & normal & wide \\
\cline { 2 - 3 } & distance ratio & & & \\
\hline Nose & distance ratio & normal & wrinkle & - \\
\hline Mouth & eccentricity & narrow & normal & widely open \\
\cline { 2 - 2 } & distance ratio & & & \\
\hline
\end{tabular}

Based on Table 1 we can read that the geometric features input are processed through the fuzzy facial inference engine and resulting the semantic facial features as output, which have both real and linguistic values. Real values as an output of defuzzification process are fed into the next fuzzy knowledge-based emotion classifier, and the linguistic values are matched with the stored fuzzy emotion rules. The process is discussed on the next section.

\section{FUZZY KNOWLEDGE-BASED FOR EMOTION RECOGNITION}

The knowledge-based fuzzy for emotion recognition consists of a set of rules to interpret emotion from facial component changes, as defined by psychologist expert Ekman [6]. Fuzzy knowledge-based is different from conventional knowledge-based which explicitly looks for rules that are contained in a rule-base. Fuzzy is more adaptive in invoking rules and resulting fuzzy degrees in the range of 0-1. Fuzzy conceptualization is the process to represent the problem in fuzzy terms. Fuzzy terms use natural linguistic or language variables that are understood by humans in everyday life. Fuzzy linguistic variables have both quantitative and qualitative value; the quantitative value is a real value and the qualitative value is the linguistic value, e.g. low, medium, high. The entire process of the fuzzy emotion recognition framework is described on the following subsections.

\section{A. Framework Design}

Fuzzy emotion recognition framework design is depicted in Fig. 4.

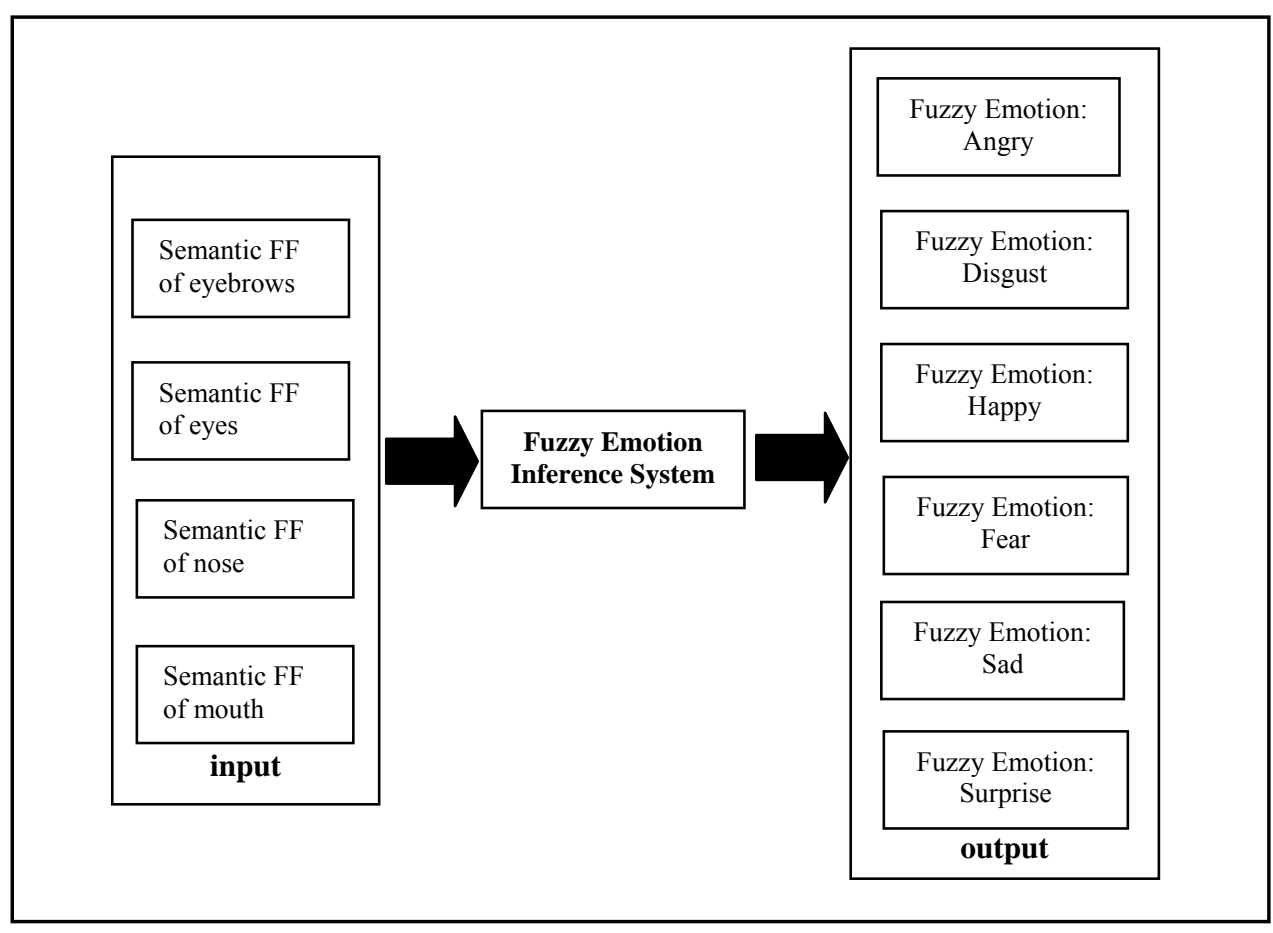

Fig. 4. Fuzzy emotion recognition framework

Input variables are four Semantic FF (Facial Features) of facial components: eyebrows, eyes, nose, and mouth. These values are obtained from the previous stage, semantic facial features extraction. Unlike any other works which design the emotion into a single output variable with basic emotion classes as the membership functions; we separate output into six fuzzy emotion variables: angry, disgust, happy, fear, sad, and surprise. This is based on a reason that basic emotion classes are not exist in a continuous interval. According to a psychologist, each 
emotion class has its own dimension as well as linguistic values, e.g. happy has three linguistic values: slight, medium, and extreme happy [2]. Hence, our proposed framework best captures the psychologist knowledge in interpreting real emotion in human face. The center rectangle in Fig. 4 is the fuzzy emotion inference engine which transforms input into output based on fuzzy emotion knowledge which is stored in the system.

\section{B. Fuzzy Emotion Inference System}

Fuzzy emotion inference system involves semantic facial features input, emotion rule base, and fuzzy emotion output. The process inside the fuzzy emotion inference system is described in Fig. 5. Initially, the semantic facial features input $x$ and $y$ are fuzzified using fuzzifier module, resulting fuzzy membership degrees $\mu(x)$ and $\mu(y)$. The fuzzy inference module consists of inference engine and fuzzy rule-base which process the fuzzy input $\mu(x)$ and $\mu(y)$ to result the fuzzy aggregate membership degree $\mu(z)$. The last step is defuzzifier module which converts the fuzzy value $\mu(z)$ into real output value $z$.

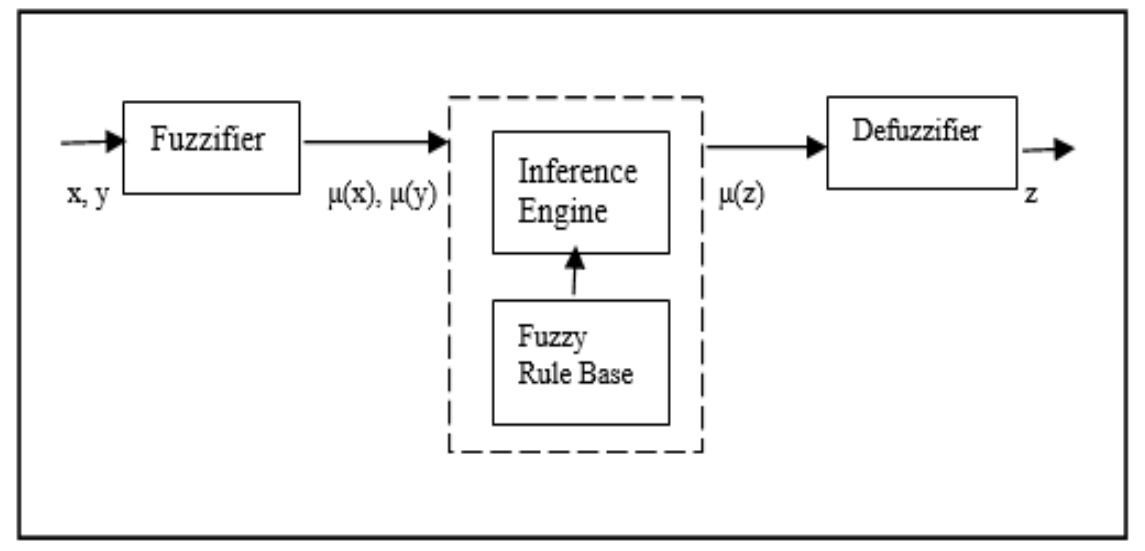

Fig. 5. Fuzzy emotion inference system process

This work implements Fuzzy Sugeno type-0 inference method with constant output based on the reason that emotion output has a categorical value, thus Fuzzy Sugeno type- 0 is suitable for such case. Constant value is set as: 1 for low; 2 for medium; and 3 for high as fuzzy output value. After the defuzzification step, the value of fuzzy emotion will be normalized in the range $[0,1]$.

Fig 6. shows the example of fuzzy input membership function for eyes. The interval value of the horizontal axis is an input value obtained from the semantic facial feature values, while the vertical axis indicates the degree of membership or fuzzy value. Three triangular membership functions are narrow, normal, and wide. The parameter setting is manually tuned based on experiments.

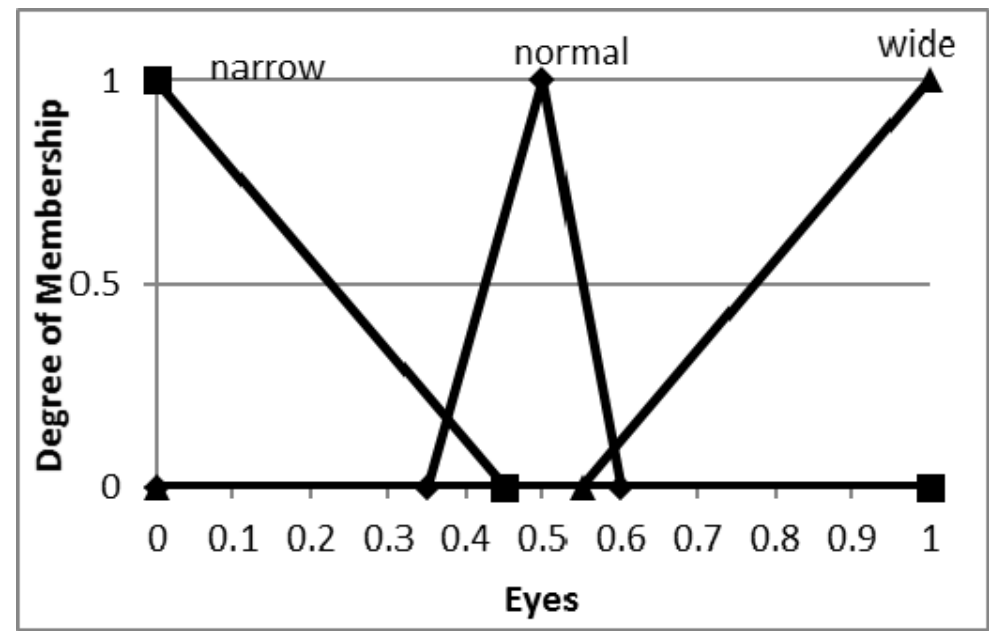

Fig. 6. Fuzzy input variable: eyes

Each fuzzy emotion knowledge-base contains a set of rules for a specific basic emotion class. Thus, there are six separated rule-base in our proposed system. As an example, fuzzy emotion rules are as follows:

1. IF eyebrow is lower AND eye is normal AND mouth is widely open THEN angry is high

2. IF eyebrow is raise AND nose is wrinkle AND mouth is narrow THEN disgust is medium

3. IF eyebrow is normal AND eye is narrow AND mouth is narrow THEN fear is low 
Fuzzy emotion works by selecting rules with match with the input from the stored rule-base and generating fuzzy emotion values. The output is based on the composition of some fuzzy rules which are invoked by some input configuration. Then, defuzzification and normalization are performed to obtain fuzzy values from each emotion class in the interval $[0,1]$, using the weighted average sum method as in equation 2.

$$
\mathrm{Z}=\frac{\sum_{i=1}^{n} \omega_{f}^{*} \widetilde{a}_{i}}{\sum_{i=1}^{n} \omega_{i}}
$$

Where $n$ is the number of input variable, $\alpha$ is the output membership degree, $z$ is the real output value in horizontal axis and $Z$ is the fuzzy emotion intensity generated by the Fuzzy Sugeno inference method.

\section{RESUlts AND Discussions}

After the recognition process, the highest fuzzy emotion value represents the highest intensity of emotion class that an image belongs to. But, the other emotions which may also occur simultaneously are not eliminated and considered as the mixed emotion at different intensities. This is the strength of fuzzy emotion to represent human real emotion. Fig. 7 shows the example of our system.

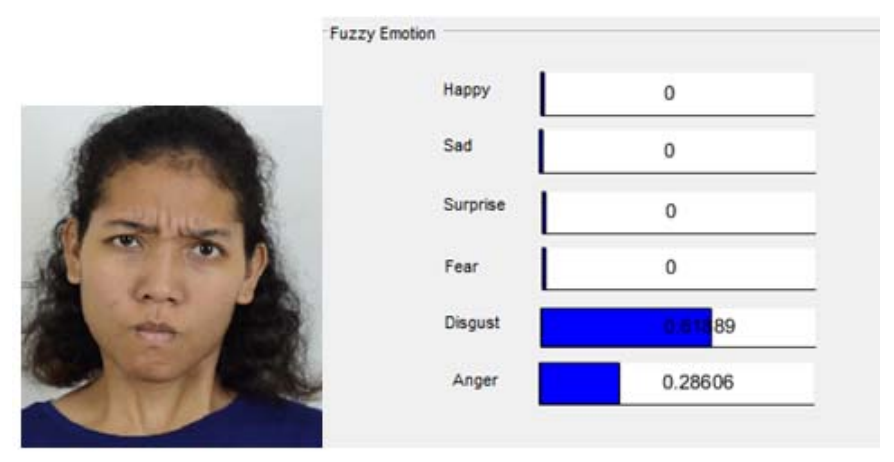

Fig. 7. Fuzzy emotion recognition output

Fig. 8 shows the output of fuzzy emotion recognition system. The visual bar next to the facial image represents the intensity of emotion which occurs with value range [0,1]. The highest value is in disgust class (with intensity 0.61889), hence it becomes the result of the classification. But there is also a slight anger in it (with intensity 0.28606 ). So, the image consists of disgust and angry emotions. Another result example where multiple emotion co-exists is displayed on Fig. 8.

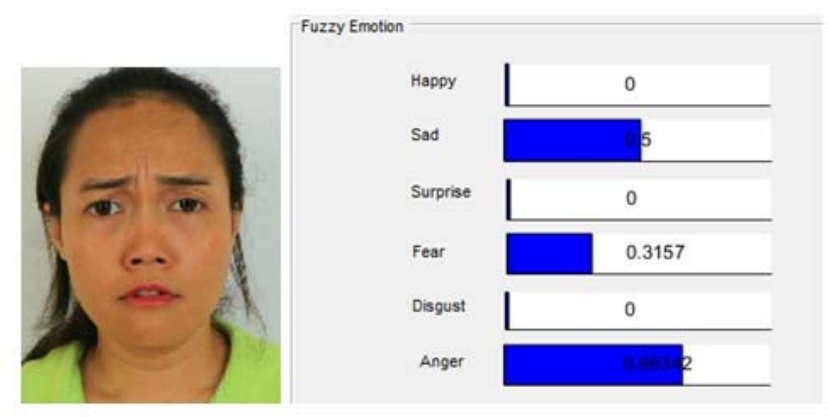

Fig. 8. Multiple emotions result

As seen on Fig. 8, three multiple emotions co-exist (sad, fear, and angry) with the highest emotion intensity is angry (0.66342). We do not remove other emotions which reveal, instead we preserve emotions as an important information contained in an image. We manage separate fuzzy knowledge based for each emotion class, hence the result resembles an emotion analysis done by a psychologist, instead of a voting mechanism as in other classifiers

We conduct a testing to measure the performance of our proposed fuzzy emotion recognition. The dataset used is IMED which consists of 270 facial images for testing with a balance number of images for six emotion classes: 45 angry, 45 disgust, 45 fear, 45 happy, 45 sad, 45 surprise. We perform the fuzzy emotion recognition and analyse the recognition results. The confusion matrix is presented on Table 2. 
TABLE 2. Confusion matrix of IMED testing

\begin{tabular}{|c|c|c|c|c|c|c|}
\hline & An & Di & Fe & $\mathrm{Ha}$ & $\mathrm{Sa}$ & $\mathrm{Su}$ \\
\hline $\mathrm{An}$ & 39 & 0 & 2 & 2 & 2 & 0 \\
\hline $\mathrm{Di}$ & 2 & 40 & 1 & 2 & 0 & 0 \\
\hline $\mathrm{Fe}$ & 3 & 1 & 39 & 2 & 0 & 0 \\
\hline $\mathrm{Ha}$ & 0 & 1 & 3 & 40 & 1 & 0 \\
\hline $\mathrm{Sa}$ & 3 & 0 & 0 & 2 & 40 & 0 \\
\hline $\mathrm{Su}$ & 1 & 0 & 0 & 2 & 1 & 41 \\
\hline
\end{tabular}

*An = angry, $\mathrm{Di}=$ disgust, $\mathrm{Fe}=$ fear, $\mathrm{Ha}=$ happy, $\mathrm{Sa}=$ sad, $\mathrm{Su}=$ surprise

Based on the confusion matrix on Table 2, the average accuracy rate is $88.519 \%$ for IMED testing. We also present the precision and recall of each emotion class and summarize the result on Table 3.

TABLE 3. Precision-Recall of IMED

\begin{tabular}{|c|c|c|}
\hline Emotion & Precision (\%) & Recall (\%) \\
\hline Angry & 86.667 & 81.250 \\
\hline Disgust & 88.889 & 95.238 \\
\hline Fear & 86.667 & 86.667 \\
\hline Happy & 88.889 & 80.000 \\
\hline Sad & 88.889 & 90.909 \\
\hline Surprise & 91.111 & 100.00 \\
\hline average & $\mathbf{8 8 . 5 1 9}$ & $\mathbf{8 9 . 0 1 1}$ \\
\hline
\end{tabular}

Result on Table 3 shows that the highest precision $(91.111 \%)$ and recall $(100 \%)$ is surprise class. While the lowest precision is angry and fear $(86.667 \%)$ and the lowest recall is happy $(80 \%)$. The average precision rate is $88.519 \%$ and the average recall rate is $89.011 \%$. We visualize the precision and recall result on a graphic in Fig. 9 to see the difference in each emotion class.

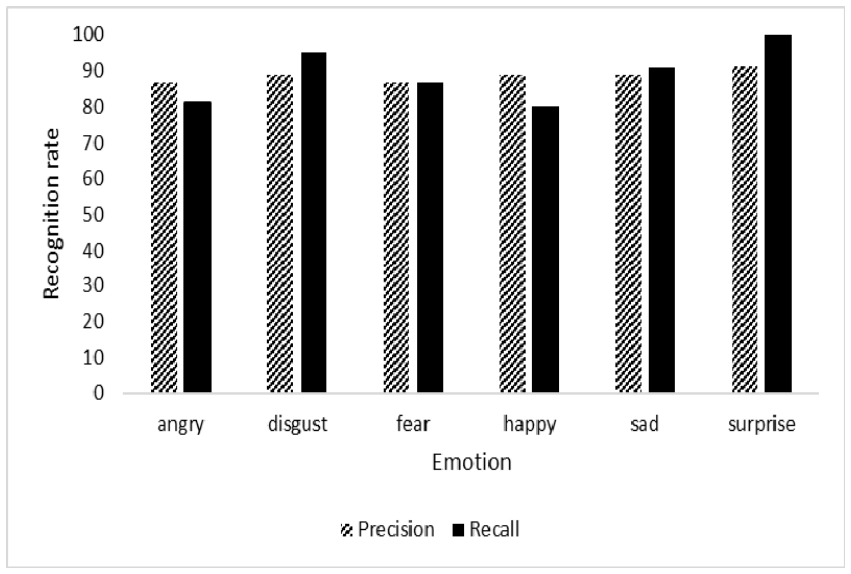

Fig. 9. Graphic of precision-recall of IMED testing

According to the graphic on Fig. 9 which shows the precision-recall of IMED testing, the difference between precision and recall is consistent in four emotion classes (disgust, fear, sad, and surprise), except for angry and happy classes where the precision is higher than the recall. The highest precision means that the system has precisely recognized the emotion class, with less misclassification class. While the highest recall means that the recognition result consists of many actual emotion classes and less false emotion class. Our proposed system results a high accuracy rate as well as precision and recall. It means that the result of the testing on IMED dataset consists more true positive values, or the system returns most of the relevant recognition result.

\section{CONCLUSION}

This paper implements fuzzy emotion recognition as a natural human emotion which tolerates the ambiguity and multiple emotion occurrence in facial expression. Fuzzy emotion is a generalization of mixed emotion which is a real human emotion displayed in a daily life interaction. The novelty of the proposed work is exposing the high-level feature extraction called semantic facial features and the fuzzy knowledge-based extracted from the psychologist knowledge and stored in a fuzzy inference engine. Thus, the recognition mimics 
the way a psychologist analyses emotion from a facial expression and facial component changes. The result of the testing using own-made Indonesian Mixed Emotion Dataset (IMED) shows that the proposed system gained high accuracy rate $88.519 \%$, precision $88.519 \%$, and recall $89.011 \%$. For the future works, the study considers optimizing the parameter of fuzzy emotion inference system to increase the recognition results.

\section{ACKNOWLEDGMENT}

The author would like to acknowledge the Ministry of research and technology and higher education of Indonesia for the research grant. The first author would like to thank Indonesia Endowment Fund (LPDP) for the $\mathrm{PhD}$ scholarship. We also deliver our appreciation to all the contributors of the development of IMED dataset.

\section{REFERENCES}

[1] P. Ekman, “Are There Basic Emotions ?,” Psychological Review, vol. 99, no. 3, pp. 550-553, 1992.

[2] P. Ekman, Emotions revealed: recognizing faces and feelings to improve communication and emotional life, 1st ed. New York: Times Books, 2003

[3] P. Lucey, J. F. Cohn, T. Kanade, J. Saragih, Z. Ambadar, and I. Matthews, "The extended Cohn-Kanade dataset (CK+): A complete dataset for action unit and emotion-specified expression,” 2010 IEEE Comput. Soc. Conf. Comput. Vis. Pattern Recognit. - Work. CVPRW 2010, no. May, pp. 94-101, 2010.

[4] M. Kamachi, M. Lyons, and J. Gyoba, "The japanese female facial expression (jaffe) database," Availble http//www. kasrl. org/jaffe. html, 1997.

[5] S. M. Mavadati, M. H. Mahoor, K. Bartlett, P. Trinh, and J. F. Cohn, "DISFA: A Spontaneous Facial Action Intensity Database," IEEE Transaction on Affective Compuing,, vol. 4, no. 2, pp. 151-160, 2013.

[6] P. Ekman and W. V Friesen, Unmasking the face: A guide to recognizing emotions from facial clues. Oxford: Prentice-Hall, 1975.

[7] V. Sathya and T. Chakravarthy, "Emotional Based Facial Expression Recognition Using Support Vector Machines," vol. 9, no. 6, International Journal of Engineering and Technology (IJET), pp. 4232-4237, 2018.

[8] D. Das and A. Chakrabarty, "Emotion recognition from face dataset using deep neural nets," Proc. 2016 Int. Symp. Innov. Intell. Syst. Appl. INISTA 2016, 2016.

[9] M. Pantic, R. Cowie, F. D’Errico, D. Heylen, M. Mehu, C. Pelachaud, I. Poggi, M. Schroeder, and A. Vinciarelli, "Social Signal Processing: The Research Agenda," Vis. Anal. Humans, pp. 511-538, 2011.

[10] A.Vinciarelli, H. Salamin, and M. Pantic, "Social Signal Processing: Understanding Social Actions through nonverbal behaviour analysis,” Proc. IEEE Comput. Soc. Conf. Comput. Vis. Pattern Recognit. Work. CVPR Work. 2009, vol. 3, no. 231287, pp. 42-49, 2009.

[11] Vinciarelli, M. Pantic, H. Bourlard, and A. Pentland, "Social signals, their function, and automatic analysis: A survey," Proc. 10th Int. Conf. Multimodal interfaces - IMCI '08, pp. 61-68, 2008.

[12] D. Y. Liliana, M. R. Widyanto, and T. Basaruddin, "Human emotion recognition based on active appearance model and semisupervised fuzzy C-means," in 2016 International Conference on Advanced Computer Science and Information Systems, ICACSIS 2016, 2017, pp. 439-445.

[13] L. Wang, R. Li, and K. Wang, "A Novel Automatic Facial Expression Recognition Method Based on AAM," Journal of Computers, vol. 9, no. 3, pp. 608-617, 2014.

[14] X. Li, Q. Ruan, Y. Jin, G. An, and R. Zhao, "Fully automatic 3D facial expression recognition using polytypic multi-block local binary patterns," Signal Processing, vol. 108, pp. 297-308, 2015.

[15] M. H. Siddiqi, R. Ali, A. M. Khan, Y. T. Park, and S. Lee, "Human Facial Expression Recognition Using Stepwise Linear Discriminant Analysis and Hidden Conditional Random Fields," IEEE Trans. Image Processing, vol. 24, no. 4, pp. 1386-1398, 2015.

[16] K. Anderson and P. W. McOwan, "A real-time automated system for the recognition of human facial expressions.," IEEE Trans. Syst. Man. Cybern. B. Cybern., vol. 36, no. 1, pp. 96-105, 2006.

[17] Z. Ming, J. Rouas, T. Shochi, Z. Ming, J. Rouas, T. Shochi, "Facial Action Units Intensity Estimation by the Fusion of Features with Multi-kernel Support Vector Machine", 11th IEEE International Conference and Workshops on Automatic Face and Gesture Recognition (FG), 2015.

[18] P. Khorrami, T. Le Paine, K. Brady, C. Dagli, and T. S. Huang, "How Deep Neural Networks Can Improve Emotion Recognition on Video Data," IEEE International Conference on Image Processing (ICIP), pp. 1-5, 2016.

[19] D. A. Pitaloka, A. Wulandari, T. Basaruddin, and D. Y. Liliana, "Enhancing CNN with Preprocessing Stage in Automatic Emotion Recognition," Procedia Computer Science, vol. 116, pp. 523-529, 2017.

[20] C. E. Izard, "Basic emotions, relations among emotions, and emotion-cognition relations.," Psychological Review, vol. 99, no. 3, pp. $561-5,1992$.

[21] S. Du, Y. Tao, and A. M. Martinez, "Compound facial expressions of emotion," Proceedings of the National Academy of Sciences, vol. 111, no. 15, pp. E1454-E1462, Apr. 2014.

[22] D. Y. Liliana, C. Basaruddin, and M. R. Widyanto, "Mix Emotion Recognition from Facial Expression using SVM-CRF Sequence Classifier," Proc. Int. Conf. Algorithms, Comput. Syst. - ICACS '17, pp. 27-31, 2017.

[23] A. Chakraborty, A. Konar, U. K. Chakraborty, and A. Chatterjee, "Emotion Recognition From Facial Expressions and Its Control Using Fuzzy Logic,” IEEE Trans. Syst. Man, Cybern. - Part A Syst. Humans, vol. 39, no. 4, pp. 726-743, 2009.

[24] A. Khanum, M. Mufti, M. Y. Javed, and M. Z. Shafiq, "Fuzzy case-based reasoning for facial expression recognition," Fuzzy Sets and System, vol. 160, pp. 231-250, 2009 .

[25] M. Ilbeygi and H. Shah-Hosseini, "A novel fuzzy facial expression recognition system based on facial feature extraction from color face images," Engineering Applications of Artificial Intelligence, vol. 25, no. 1, pp. 130-146, 2012.

[26] K. Lekdioui, R. Messoussi, Y. Ruichek, Y. Chaabi, and R. Touahni, "Facial decomposition for expression recognition using texture/shape descriptors and SVM classifier," Signal Processing and Image Communication, vol. 58, no. July, pp. 300-312, 2017.

[27] J. Chen, D. Chen, Y. Gong, M. Yu, K. Zhang, and L. Wang, "Facial expression recognition using geometric and appearance features," Proc. 4th Int. Conference on. Internet Multimedia and Computer Service - ICIMCS '12, p. 29, 2012.

[28] T. F. Cootes, G. J. Edwards, and C. J. Taylor, “Active appearance models," IEEE Trans. Pattern Analysis and. Machine Intelligence, vol. 23, no. 6, pp. 681-685, Jun. 2001.

[29] G. Tzimiropoulos and M. Pantic, "Optimization Problems for Fast AAM Fitting in-the-Wild," in 2013 IEEE International Conference on Computer Vision, 2013, pp. 593-600.

[30] J. Kumari, R. Rajesh, and K. M. Pooja, "Facial Expression Recognition: A Survey,” Procedia Computer Science, vol. 58, pp. 486-491, 2015. 
[31] Xinbo Gao, Ya Su, Xuelong Li, and Dacheng Tao, "A Review of Active Appearance Models," IEEE Transaction on System, Man, Cybernetics. Part C (Applications Rev., vol. 40, no. 2, pp. 145-158, 2010.

[32] D. Y. Liliana, M. R. Widyanto, and T. Basaruddin, "Geometric Facial Components Feature Extraction for Facial Expression Recognition," in 2018 International Conference on Advanced Computer Science and Information Systems (ICACSIS), 2018, pp. 391396.

\section{AUTHOR PROFILE}

Dewi Yanti Liliana is a Ph.D student at Faculty of Computer Science, Universitas Indonesia. She is also a lecturer at State Polytechnic of Jakarta. She received her master's degree on Computer Science from Universitas Indonesia, and Bachelor of Computer Science from Sepuluh Nopember Institute of Technology, Indonesia. Her research topic is focusing on emotion recognition from facial expression analysis through computer vision and machine learning modeling.

T. Basaruddin is a Professor of Computer Science at Universitas Indonesia. He received his PhD and master's degree from Manchester University, UK, and bachelor's degree on mathematics from Gajah Mada University, Indonesia. His research interests are on Social Signal Processing, Machine learning, Numerical Analysis, and Computational Intelligence.

M. Rahmat Widyanto is a Doctor of Computer Science at Universitas Indonesia. He received his $\mathrm{PhD}$ and master's degree from Tokyo Institute of Technology, Japan, and bachelor's degree on Computer Science from Universitas Indonesia. His research interests are on Artificial Intelligence, Soft Computing, and Fuzzy System. 